\title{
Reduction in daily hydrocortisone dose improves bone health in primary adrenal insufficiency
}

\author{
Julia Schulz' ${ }^{1}$, Kathrin R Frey ${ }^{2}$, Mark S Cooper ${ }^{3}$, Kathrin Zopf ${ }^{1}$, Manfred Ventz', \\ Sven Diederich ${ }^{4}$ and Marcus Quinkler ${ }^{1,5}$ \\ ${ }^{1}$ Department of Clinical Endocrinology, Charité Campus Mitte, Charité University Medicine Berlin, Berlin, Germany, \\ ${ }^{2}$ Endocrine and Diabetes Unit, Department of Medicine I, University Hospital, University of Würzburg, Würzburg, \\ Germany, ${ }^{3}$ Adrenal Steroid Group, ANZAC Research Institute, Concord Repatriation General Hospital, Hospital \\ Road, Concord Hospital, Concord, New South Wales 2139, Australia, ${ }^{4}$ Endokrinologikum, Berlin, Germany and \\ ${ }^{5}$ Endocrinology in Charlottenburg, Stuttgarter Platz 1, 10627 Berlin, Germany
}

Correspondence should be addressed to M Quinkler

Email

marcus.quinkler@t-online.de

\begin{abstract}
Objective: Individuals with primary adrenal insufficiency (PAI) or congenital adrenal hyperplasia (CAH) receive life-long glucocorticoid (GC) replacement therapy. Current daily GC doses are still higher than the reported adrenal cortisol production rate. This GC excess could result in long-term morbidities such as osteoporosis. No prospective trials have investigated the long-term effect of GC dose changes in PAI and CAH patients.

Methods: This is a prospective and longitudinal study including 57 subjects with PAI (42 women) and 33 with CAH ( 21 women). Bone mineral density (BMD) was measured by dual energy $\mathrm{X}$-ray absorptiometry at baseline and after 2 years. Subjects were divided into three groups (similar baseline characteristics) depending on changes in daily hydrocortisone equivalent dose (group 1: unchanged $25.2 \pm 8.2 \mathrm{mg}$ (mean \pm s.D., $n=50$ ); group 2: increased $18.7 \pm 10.3$ to $25.9 \pm 12.0 \mathrm{mg}$ ( $n=13$ ); group 3: decreased $30.8 \pm 8.5$ to $21.4 \pm 7.2 \mathrm{mg}(n=27))$.

Results: Subjects in group 1 showed normal lumbar and femoral $Z$-scores which were unchanged over time. Group 2 subjects showed a significant decrease in femoral neck $Z$-scores over time $(-0.15 \pm 1.1$ to $-0.37 \pm 1.0(P<0.05))$, whereas group 3 subjects showed a significant increase in lumbar spine and hip Z-scores (L1-L4: $-0.93 \pm 1.2$ to $-0.65 \pm 1.5(P<0.05)$; total hip: $-0.40 \pm 1.0$ to $-0.28 \pm 1.0(P<0.05))$. No changes in BMl over time were seen within any group. Reduction in GC dose did not increase the risk of adrenal crisis.

Conclusion: This study demonstrates for the first time that cautious reduction in hydrocortisone equivalent doses leads to increases in BMD, whereas dose increments reduced BMD. These data emphasize the need for the lowest possible GC replacement dose in Al patients to maintain health and avoid long-term adverse effects.
\end{abstract}

\section{Introduction}

Glucocorticoids (GCs) are widely used for their immunosuppressive and anti-inflammatory effects. However, the main adverse effects of this pharmacotherapy are GC-induced osteoporosis, diabetes mellitus and obesity. These features appear strongly dose and time dependent. However, endogenous GCs are the key regulators of mesenchymal cell differentiation and normal bone development at physiological concentrations. In states of adrenal insufficiency, such as primary adrenal insufficiency (PAI) (c) 2016 European Society of Endocrinology Printed in Great Britain and congenital adrenal hyperplasia (CAH), GCs are given in much lower doses as hormone replacement therapy. However, the daily intake of GCs in PAI and CAH still tends to be more than the total daily endogenous cortisol secretion seen in healthy subjects $(5-10 \mathrm{mg}$ of cortisol per $\mathrm{m}^{2}$ of body surface area per day) (1).

Most studies on bone mineral density (BMD) in PAI and $\mathrm{CAH}$ patients are limited by small patient numbers and heterogeneous populations and methods, especially

Published by Bioscientifica Ltd. 
with respect to age and GC regimes. These studies have shown inconsistent findings relating to BMD, disease duration, GC type and daily dose. Some studies reported reduced BMD in all PAI patients $(2,3)$, some only in postmenopausal women $(4,5)$ or only in men $(6,7)$, whereas other studies found no significant differences in BMD in either gender $(8,9,10)$. In CAH patients some studies reported no significant differences in BMD between patients with CAH and controls (10, 11, 12, 13, $14,15,16)$, whereas others found reduced BMD in all or some subpopulations of CAH patients $(17,18,19,20$, $21,22,23,24)$.

Until now all studies in this area have had a crosssectional design. No longitudinal study examining the effect of decreasing or increasing GC doses on BMD in PAI or CAH patients has been performed to date.

The aim of this study was to prospectively examine the effect of decreasing or increasing the daily GC dose on BMD in PAI and CAH patients followed over a period of 2 years.

\section{Subjects and methods}

\section{Design and subjects}

This was a 2-year prospective, single-centre study including patients from one university endocrine outpatient clinic. Inclusion criteria were PAI or CAH verified by review of the medical records. Patients under the age of 18 years, patients with diagnosed bone metastasis, adrenal insufficiency due to long-term pharmacological GC treatment, adrenocortical carcinoma and patients with less than 12 months duration of disease were excluded. The study protocol was approved by the local ethics committee (EA1/027/10) and all patients gave written informed consent prior to participation. A total of 100 patients with PAI, and 50 patients with CAH were asked to participate in the study. Thirty patients declined to take part, and thus 120 patients were included in the study.

At study enrolment and after 2 years (follow-up), BMD was assessed by dual energy X-ray absorptiometry (DXA) scan, and all clinical data, including the hormone replacement therapies and changes during the 2 years, were documented. The patients were seen every 4-6 months by two experienced endocrinologists. The endocrinologists had the aim to optimize GC replacement therapy according to the best clinical practice guidelines $(25,26,27)$ : the monitoring of GC replacement predominantly relied on the clinical assessment including the search for symptoms and signs of over- or underreplacement. In addition, a detailed questioning regarding the daily intake of medication, the perceived general feeling of energy levels and 'get up and go', mental concentration, daytime somnolence and changes in pigmentation was performed during the clinical visits (25). Also information regarding low points or dips in energy during the day was asked by the endocrinologists (25). All patients received structured education regarding dose adaptation in the event of intercurrent illness and management of adrenal crisis every 6 months.

At the end of the study the patients were divided into three groups based on a criteria predefined before the study commenced: group 1 with unchanged daily hydrocortisone equivalent doses during the study time, group 2 with increased and group 3 with decreased daily hydrocortisone equivalent doses at follow-up visit compared with the baseline.

Of the 120 patients included, 30 patients had an incomplete data set after 2 years or refused to have a DXA scan at follow-up, and therefore were excluded. For final analysis there were 57 patients suffering from PAI (42 women; mean age 54.1 years) and 33 patients with CAH (21 women; mean age 39.5 years; salt-wasting $n=15$, simple-virilizing $n=11$, late-onset $n=6,11 \beta$-hydroxylase deficiency $n=1$ ). The underlying cause of PAI was isolated autoimmune adrenal insufficiency $(n=17 ; 29.8 \%)$, autoimmune polyendocrine syndrome type II (=APS2; $n=24 ; 42.1 \%)$, bilateral adrenalectomy $(n=12 ; 21.1 \%$; due to Cushing's disease, bilateral pheochromocytoma, or renal cell carcinoma metastasis; operation $>5$ years ago), unilateral adrenalectomy with insufficient remaining adrenal function $(n=1 ; 1.75 \%)$, tuberculosis $(n=2 ; 3.5 \%)$ or triple A syndrome $(n=1 ; 1.75 \%)$.

For analysis the dose of GC was converted into milligrams of hydrocortisone equivalent $(1 \mathrm{mg}$ dexamethasone $=70 \mathrm{mg}$ hydrocortisone, $1 \mathrm{mg}$ prednisolone $=$ $6 \mathrm{mg}$ hydrocortisone) $(28,29)$. The daily hydrocortisone equivalent intake was corrected for body surface area. The patient management data at baseline and follow-up are presented in Table 1.

\section{Measurements}

Blood was drawn for measurement at baseline and 2 years later at follow-up for the following parameters: serum calcium (Ca; Roche Diagnostics $\mathrm{GmbH}$ ), phosphorus (P; Roche Diagnostics $\mathrm{GmbH}$ ), alkaline phosphatase (AP; Roche Diagnostics $\mathrm{GmbH}$ ), parathyroid hormone (PTH; Elecsys-PTH, Roche Diagnostics GmbH), 
Table 1 Management data of 90 patients with primary adrenal insufficiency (PAI) or congenital adrenal hyperplasia (CAH) with a complete data set at baseline and follow-up. Daily fludrocortisone ranged between 0.05 and $0.15 \mathrm{mg}$. Data are presented as mean \pm S.D. or as $n(\%)$.

\begin{tabular}{l}
\hline \\
Sex (male/female) \\
Daily HC-equivalent dose in $\mathrm{mg}$ (range) \\
GC-dose per body surface in $\mathrm{mg} / \mathrm{m}^{2}$ (range) \\
GC type, $n$ (\%) \\
Hydrocortisone \\
Prednisolone \\
Dexamethasone \\
Prednisolone + dexamethasone \\
Hydrocortisone + dexamethasone \\
Hydrocortisone + prednisolone \\
Fludrocortisone therapy, $n$ (\%) \\
Vitamin D therapy, $n(\%)$ \\
DHEA therapy, $n(\%)$
\end{tabular}

\begin{tabular}{c}
\hline \\
\hline Baseline \\
\hline $15 / 42$ \\
$25.3 \pm 6.5(10-45)$ \\
$13.9 \pm 3.9(5.4-24.6)$ \\
$39(68.4 \%)$ \\
$18(31.6 \%)$
\end{tabular}

PAI

\begin{tabular}{c} 
Follow-up \\
\hline $15 / 42$ \\
$25.0 \pm 7.0(15-50)$ \\
$13.8 \pm 4.5(7.4-33.2)$
\end{tabular}

$41(71.9 \%)$

$16(28.1 \%)$

$29(50.9 \%)$

$14(33.3 \%$ of women)

\begin{tabular}{|c|c|}
\hline \multicolumn{2}{|c|}{ CAH } \\
\hline Baseline & Follow-up \\
\hline $12 / 21$ & $12 / 21$ \\
\hline $27.1 \pm 12.9(0-60)$ & $22.7 \pm 11.0(0-60)$ \\
\hline $15.9 \pm 7.8(0-33.8)$ & $13.3 \pm 6.7(0-36.9)$ \\
\hline $11(34.4 \%)$ & $14(43.8 \%)$ \\
\hline $3(9.4 \%)$ & $4(12.5 \%)$ \\
\hline $5(15.6 \%)$ & $3(9.4 \%)$ \\
\hline $3(9.4 \%)$ & $2(6.3 \%)$ \\
\hline $9(28.1 \%)$ & $7(21.9 \%)$ \\
\hline $1(3.1 \%)$ & $2(6.3 \%)$ \\
\hline $17(51.5 \%)$ & $17(51.5 \%)$ \\
\hline $4(12.1 \%)$ & $22(66.7 \%)$ \\
\hline
\end{tabular}

HC, hydrocortisone; GC, glucocorticoid.

and 25-hydroxyvitamin D3 (25(OH)D3; IDS-ISYS immunoassay, Immunodiagnostic Systems (IDS) GmbH, Frankfurt, Germany).

BMD was measured using DXA (GE Lunar DPX-NT, Madison, WI, USA) at the femoral neck, greater trochanter, total hip and lumbar spine (L1-L4) according to the manufacturer's standard scan and positioning protocols. Spine phantom quality control scans were acquired on a daily basis during the study. Phantom data were entered into a Shewhart chart with a previously established baseline (the mean of ten initial control scans) and was always within $\pm 1.5 \%$ from the initial mean. The precision error was s.D. $0.004 \mathrm{~g} / \mathrm{cm}^{2}, \mathrm{CV} 0.414 \%$, or below. The scans were analyzed using GE-Lunar Software version enCORE 2004 (GE, Madison, WI, USA) and the results from the BMD measurements were transformed into $Z$-scores using sex- and age-matched reference data provided by the manufacturer originating from an American population.

To compare patients' complaints and fatigue over the study period, patients were asked to complete the recently developed Addison's disease-specific questionnaire (AddiQoL) $(30,31)$ at baseline and follow-up. Of the 90 included patients, 72 patients $(72.2 \%)$ had a complete questionnaire at baseline and follow-up. The recently developed AddiQoL for patients with PAI initially included 36 questions (30) and was validated and reduced to a 30 item questionnaire (31). Items that are positive had scores from 1 to 6 ; negative health-related quality of life (HRQoL) statements were reversed for questionnaire scoring (6 to 1$)$. The scoring was converted to points:
$6=4$ points; 5 and $4=3$ points; 3 and $2=2$ points; and $1=1$ point. The algebraic sum of points was calculated. Higher points indicate a higher level of HRQoL. The fatigue subscale, which consists of questions 1-5 and 23, 26 and 27 was also calculated.

Table 2 Clinical data and bone mineral density (BMD) of the 90 included patients with complete data sets at baseline and follow-up (after $28.7 \pm 5.6$ months). $Z$-scores represent age and gender-adjusted SDS.

\begin{tabular}{|c|c|c|}
\hline & Baseline & Follow-up \\
\hline$n(\mathrm{PAI} / \mathrm{CAH})$ & $90(57 / 33)$ & $90(57 / 33)$ \\
\hline Sex (male/female) & $27 / 63$ & $27 / 63$ \\
\hline Age (year) & $48.3 \pm 15.1$ & $50.6 \pm 15.1^{*}$ \\
\hline $\mathrm{BMI}\left(\mathrm{kg} / \mathrm{m}^{2}\right)$ & $26.3 \pm 4.6$ & $26.4 \pm 4.6$ \\
\hline Duration of disease (year) & $22.3 \pm 14.1$ & $24.3 \pm 14.1^{*}$ \\
\hline Daily HC-equivalent dose (mg) & $26.0 \pm 9.4$ & $24.1 \pm 8.7^{\dagger}$ \\
\hline GC-dose per body surface $\left(\mathrm{mg} / \mathrm{m}^{2}\right)$ & $14.6 \pm 5.7$ & $13.6 \pm 5.4$ \\
\hline Calcium (2.15-2.65 mmol/l) & $2.32 \pm 0.1$ & $2.41 \pm 0.1 *$ \\
\hline Phosphorus (0.8-1.5 mmol/l) & $0.95 \pm 0.2$ & $0.93 \pm 0.2$ \\
\hline $\begin{array}{l}\text { Alkaline phosphatase } \\
\qquad(35-104 \mathrm{U} / \mathrm{l})\end{array}$ & $58.3 \pm 16.3$ & $59.2 \pm 16.4$ \\
\hline $\begin{array}{l}\text { Parathyroid hormone } \\
\quad(11-67 \mathrm{pg} / \mathrm{ml})\end{array}$ & $36.6 \pm 20.8$ & $36.2 \pm 23.0$ \\
\hline $\begin{array}{l}\text { 25-hydroxyvitamin } D_{3} \\
\quad(50-250 \mathrm{nmol} / \mathrm{l})\end{array}$ & $58.8 \pm 21.1$ & $82.3 \pm 26.3^{*}$ \\
\hline Z-score lumbar spine (L1-L4) & $-0.42 \pm 1.2$ & $-0.31 \pm 1.3^{\dagger}$ \\
\hline Z-score femoral neck & $-0.21 \pm 1.1$ & $-0.21 \pm 1.1$ \\
\hline$Z$-score greater trochanter & $-0.01 \pm 1.1$ & $-0.02 \pm 1.2$ \\
\hline Z-score total hip & $-0.14 \pm 1.0$ & $-0.10 \pm 1.1$ \\
\hline
\end{tabular}

PAl, primary adrenal insufficiency; $\mathrm{CAH}$, congenital adrenal hyperplasia; $\mathrm{HC}$, hydrocortisone; GC, glucocorticoid. Means \pm s.D. ${ }^{*} P<0.001$ vs baseline and ${ }^{\dagger} P<0.05$ vs baseline. 


\section{Statistical analysis}

BMD is expressed by $Z$-scores, which represent age and gender-adjusted SDS, which are presented with 95\% CI, and derived from quality controls performed according to the manufacturer's standards, as described in the manuals of standard operating procedures. CI different from zero indicates $Z$-scores statistically different from the reference population. Statistical analyses of paired samples were performed using the paired $t$-test or the Wilcoxon signedrank test depending on normality distribution. Statistical analyses of differences between groups were performed using the one-way ANOVA, Kruskal-Wallis ANOVA and $\chi^{2}$-test where appropriate. Post-hoc pairwise comparison between groups was tested for significance using Bonferroni test to correct for multiple comparisons. In addition, Spearman's correlation was used to assess the association between BMD and daily hydrocortisone equivalent dose and daily hydrocortisone equivalent dose per body surface. No further multivariable adjustment was performed given that baseline values were similar. Analyses were performed using the statistical software package SPSS, version 22.0 (SPSS, Inc.). Significance was accepted if $P<0.05$.

\section{Results}

Clinical data and BMD of the 90 included patients with complete data sets at baseline and follow-up are presented in Table 2. The whole cohort showed a significant decrease in HC equivalent doses and a small but significant increase in $Z$-scores at the lumbar site during the study period (Table 2). Analysis depending on the change in daily $\mathrm{HC}$ equivalent dose at follow-up showed that patients of group 2 (increased GC dose) were significantly younger and leaner than patients of group 1 (unchanged GC dose) (Table 3). Per definition of the groups, group 2 received significant lower and group 3 significantly higher daily hydrocortisone equivalent doses at baseline. However, there was no difference in duration of disease between the groups.

Importantly no changes in BMI were observed during the study period. Also serum levels of phosphorus, alkaline phosphatase and PTH stayed stable. However, serum calcium levels significantly increased in all three groups during the study period, in addition to a higher 25-hydroxyvitamin D3 status (Table 3). This was most likely due to the higher prescription rate of calcium/ vitamin D medication during the study period. However, the percentage of patients receiving calcium/vitamin D medication at follow-up was not significant different among the three groups.

Patients of group 1 showed unchanged normal $Z$-scores of lumbar and femoral areas over time. Patients of group 2 showed a significant decrease in $Z$-scores of femoral neck over time $(-0.15 \pm 1.1$ to $-0.37 \pm 1.0(P<0.05)$, mean \pm s.D.), whereas patients of group 3 showed a significant increase in $Z$-scores at lumbar and femoral sites

Table 3 Clinical data and bone mineral density (BMD) at baseline and follow-up after $28.7 \pm 5.6$ months depending on change in daily $\mathrm{HC}$ equivalent dose at follow-up in patients with primary adrenal insufficiency (PAI) or congenital adrenal hyperplasia (CAH).

\begin{tabular}{l}
\hline \\
\hline$n$ (PAl/CAH) \\
Sex (male/female) \\
Age (year) \\
BMI $\left(\mathrm{kg} / \mathrm{m}^{2}\right)$ \\
Duration of disease (year) \\
Daily HC-equivalent dose $(\mathrm{mg})$ \\
GC-dose per body surface $\left(\mathrm{mg} / \mathrm{m}^{2}\right)$ \\
Calcium $(2.15-2.65 \mathrm{mmol} / \mathrm{l})$ \\
Phosphorus $(0.8-1.5 \mathrm{mmol} / \mathrm{l})$ \\
Alkaline phosphatase $(35-104 \mathrm{U} / \mathrm{l})$ \\
Parathyroid hormone $(11-67 \mathrm{pg} / \mathrm{ml})$ \\
25-hydroxyvitamin $\mathrm{D}_{3}(50-250 \mathrm{nmol} / \mathrm{l})$ \\
Z-score lumbar spine $(\mathrm{L} 1-\mathrm{L} 4)$ \\
Z-score femoral neck \\
Z-score greater trochanter \\
Z-score total hip
\end{tabular}

\begin{tabular}{ccc}
\hline HC equivalent dose unchanged \\
\cline { 1 - 2 } Baseline & & Follow-up \\
\cline { 1 - 1 } $50(38 / 12)$ & & $50(38 / 12)$ \\
$15 / 35$ & & $15 / 35$ \\
$52.9 \pm 15.1$ & & $55.2 \pm 15.2^{*}$ \\
$26.8 \pm 4.1$ & & $26.8 \pm 4.1$ \\
$20.3 \pm 14.5$ & & $22.2 \pm 14.4^{*}$ \\
$25.2 \pm 8.2$ & & $25.2 \pm 8.2$ \\
$13.9 \pm 4.4$ & & $13.9 \pm 4.4$ \\
$2.32 \pm 0.1$ & & $2.40 \pm 0.1 *$ \\
$0.93 \pm 0.2$ & & $0.91 \pm 0.2$ \\
$57.8 \pm 13.7$ & & $60.5 \pm 15.7$ \\
$35.0 \pm 20.4$ & & $35.5 \pm 15.2$ \\
$61.5 \pm 21.3$ & & $87.5 \pm 25.2^{*}$ \\
$-0.19 \pm 1.2$ & $-0.12 \pm 1.2$ \\
$-0.02 \pm 1.2$ & $-0.01 \pm 1.2$ \\
$0.11 \pm 1.2$ & $0.10 \pm 1.3$ \\
$0.00 \pm 1.1$ & $0.01 \pm 1.1$ \\
\hline
\end{tabular}

\begin{tabular}{ccc}
\hline HC equivalent dose increased \\
\cline { 1 - 1 } Baseline & & Follow-up \\
\cline { 1 - 1 } $13(6 / 7)$ & & $13(6 / 7)$ \\
$3 / 10$ & & $3 / 10$ \\
$38.7 \pm 13.2^{\dagger}$ & & $41.2 \pm 12.9^{*}$ \\
$23.2 \pm 2.0^{\dagger}$ & & $23.7 \pm 2.3^{\ddagger}$ \\
$25.4 \pm 12.3$ & & $27.7 \pm 12.2^{*}$ \\
$18.7 \pm 10.3^{\dagger}$ & & $25.9 \pm 12.0^{*}$ \\
$11.9 \pm 7.9$ & & $16.2 \pm 9.2^{*}$ \\
$2.30 \pm 0.1$ & & $2.47 \pm 0.1^{*}$ \\
$0.98 \pm 0.2$ & & $0.94 \pm 0.2$ \\
$52.6 \pm 12.9$ & $55.3 \pm 17.2$ \\
$41.8 \pm 22.7$ & & $47.3 \pm 55.9$ \\
$56.1 \pm 25.1$ & $74.6 \pm 34.7$ \\
$-0.24 \pm 1.1$ & $-0.32 \pm 1.3$ \\
$-0.15 \pm 1.1$ & $-0.37 \pm 1.0^{\S}$ \\
$0.04 \pm 1.0$ & $-0.17 \pm 1.0$ \\
$-0.16 \pm 0.7$ & $-0.34 \pm 0.6$ \\
\hline
\end{tabular}

\begin{tabular}{ccc}
\hline HC equivalent dose decreased \\
\cline { 1 - 2 } Baseline & & Follow-up \\
\cline { 1 - 1 } $27(13 / 14)$ & & $27(13 / 14)$ \\
$9 / 18$ & & $9 / 18$ \\
$44.4 \pm 12.8$ & & $46.8 \pm 12.8^{*}$ \\
$26.7 \pm 5.7$ & & $27.1 \pm 5.9$ \\
$24.4 \pm 14.2$ & & $26.5 \pm 14.2^{*}$ \\
$30.8 \pm 8.5^{\dagger}$ & & $21.4 \pm 7.2^{*}$ \\
$17.3 \pm 5.7^{\dagger}$ & & $11.8 \pm 4.1^{*}$ \\
$2.32 \pm 0.1$ & & $2.41 \pm 0.1^{\S}$ \\
$0.96 \pm 0.2$ & & $0.97 \pm 0.2$ \\
$60.1 \pm 21.8$ & & $57.7 \pm 16.7$ \\
$36.1 \pm 23.4$ & & $33.4 \pm 12.8$ \\
$56.7 \pm 19.4$ & & $78.3 \pm 24.3^{*}$ \\
$-0.93 \pm 1.2$ & & $-0.65 \pm 1.5^{\S}$ \\
$-0.58 \pm 0.9$ & & $-0.50 \pm 1.0$ \\
$-0.25 \pm 1.0$ & & $-0.14 \pm 1.1$ \\
$-0.40 \pm 1.0$ & & $-0.28 \pm 1.0^{\S}$
\end{tabular}

$Z$-scores represent age and gender-adjusted SDS. Means \pm s.D. ${ }^{*} P<0.001$ vs baseline, ${ }^{\dagger} P<0.05$ vs 'dose unchanged' baseline, ${ }^{\ddagger} P<0.05$ vs 'dose unchanged' follow-up and ${ }^{\S} P<0.05$ vs baseline. 
(L1-L4: $-0.93 \pm 1.2$ to $-0.65 \pm 1.5(P<0.05)$; total hip: $-0.40 \pm 1.0$ to $-0.28 \pm 1.0(P<0.05))$ (Table 3$)$.

The daily HC equivalent dose and the daily GC dose per body surface negatively correlated with $Z$-scores of greater trochanter at baseline $(R=-0.291, P=0.007$; $R=-0.244, \quad P=0.024$ respectively) and follow-up ( $R=-0.326, P=0.002 ; R=-0.291, P=0.007$ respectively) (Fig. 1). Furthermore, at baseline daily $\mathrm{HC}$ equivalent dose negatively correlated with $Z$-scores of the total hip ( $R=-0.233, P=0.034)$, and lumbar regions (L1-L3: $R=-0.259, P=0.014)$.

Changes in daily $\mathrm{HC}$ equivalent dose after $28.7 \pm 5.6$ months negatively correlated with changes in $Z$-score at femoral neck $(R=-0.279, P=0.01)$, at total hip
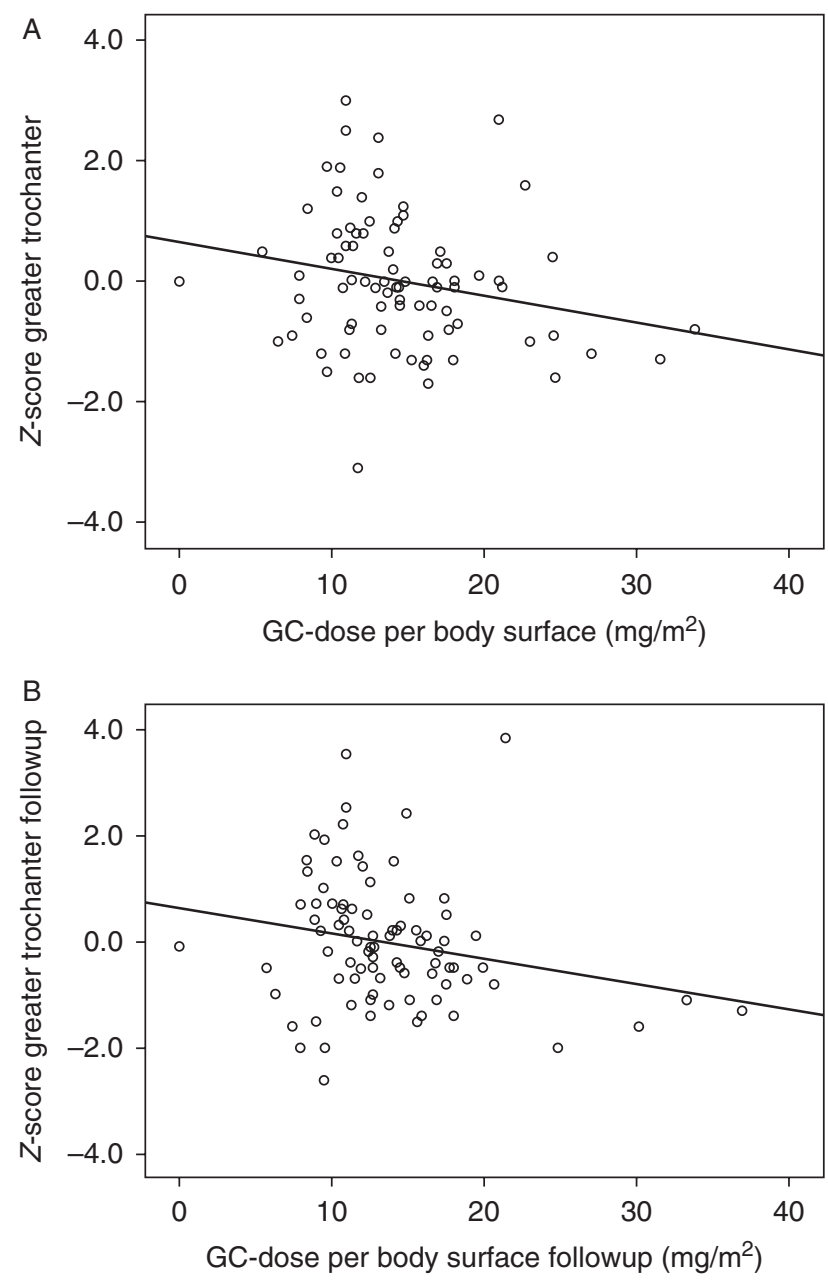

Figure 1

Correlation between Z-score greater trochanter and daily glucocorticoid-dose per body surface at baseline $(A)$ and follow-up (B).

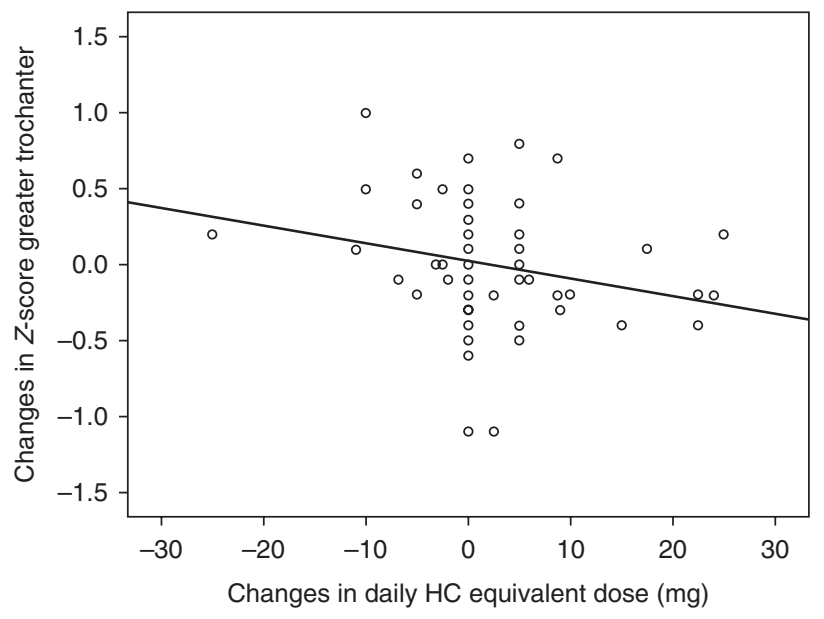

Figure 2

Correlation between changes in daily $\mathrm{HC}$ equivalent dose and changes in Z-score at greater trochanter after $28.7 \pm 5.6$ months.

$(R=-0.305, \quad P=0.005)$ and at greater trochanter $(R=-0.284, P=0.008)$ (Fig. 2).

Compared with PAI patients on hydrocortisone medication, PAI patients on prednisolone medication showed significant lower $Z$-scores at femoral neck $(0.15 \pm 1.2$ vs $-0.57 \pm 0.7(P<0.05)$ respectively), greater trochanter $(0.27 \pm 1.2$ vs $-0.71 \pm 0.7(P<0.05))$, and total hip $(0.18 \pm 1.1$ vs $-0.69 \pm 0.6(P<0.05))$ at baseline, as well as at greater trochanter $(0.24 \pm 1.3$ vs $-0.61 \pm 0.8$ $(P<0.05))$ and total hip $(0.18 \pm 1.2$ vs $-0.53 \pm 0.7$ $(P<0.05))$ at follow-up. No significant differences in $Z$-scores were seen at the lumbar spine. In $\mathrm{CAH}$ patients no significant differences in $Z$-scores were seen between $\mathrm{CAH}$ patients with $\mathrm{HC}$ and/or prednisolone medication $(n=15)$ compared with CAH patients with any dexamethasone medication $(n=17)$.

Due to safety reasons we documented all adrenal crises that occurred during the study period. In group 1 (unchanged GC dose) we documented five adrenal crises in 104.3 patient years resulting in a frequency of 4.79 crisis per 100 patient years. In group 2 (increased GC dose) we observed three adrenal crises in 32.9 patient years resulting in a frequency of 9.11 crisis per 100 patient years. Finally, in group 3 (decreased GC dose) we documented no adrenal crisis in 52.6 patient years resulting in a calculated frequency of 0 crises per 100 patient years.

Group 1 (unchanged GC dose) had a mean AddiQoL score of $83.2 \pm 13.5$ at baseline and $82.5 \pm 14.3$ at follow-up, and mean Fatigue scores of $22.0 \pm 4.4$ and $21.5 \pm 5.2$ respectively. In group 2 (increased GC dose) the mean 
AddiQoL score was $76.2 \pm 21.5$ at baseline and $78.3 \pm 12.2$ at follow-up, with mean Fatigue scores of $19.2 \pm 6.7$ and $21.0 \pm 4.2$ respectively. Finally, group 3 (decreased GC dose) had also stable mean AddiQoL scores of $85.3 \pm 10.5$ at baseline and $85.8 \pm 12.7$ at follow-up, with mean Fatigue scores of $22.8 \pm 3.5$ and $22.7 \pm 4.1$ respectively. There were no statistically significant changes in AddiQoL or Fatigue scores.

\section{Discussion}

A major concern of GC therapy using pharmacological doses is the detrimental effect on bone, which is known to be strongly dose dependent. The GC doses traditionally used in hormone replacement therapy are much lower and have even decreased further over the last two decades from an average of 30 to $20 \mathrm{mg}$ daily hydrocortisone. At doses of $30 \mathrm{mg}$ daily hydrocortisone negative effects on bone have been observed and a dose-response relationship was described (3). However, at low doses of $20 \mathrm{mg}$ daily hydrocortisone no differences in BMD could be observed compared with healthy controls (10). This strongly suggests that the previously seen detrimental effects of GC replacement therapy in PAI were primarily a consequence of supraphysiological GC exposure.

This study is the first prospective and longitudinal study investigating the impact of changes in GC doses on BMD over a longer period. Despite BMD being in the normal range and no cases of osteoporosis present, we clearly could detect a positive effect on BMD when the daily GC dose was reduced. On the other hand, we observed a clear decrease in BMD when the GC dose was increased. This resulted in a significant correlation between GC doses and BMD even in this range of contemporary low dose GC replacement. We estimate that the average increase in BMD at the lumbar spine seen in our patients with reduced GC doses would suggest a reduction of fracture risk of approximately 30\% (32). This figure may be an underestimate of the fracture risk reduction since it is currently thought that GCs can increase fracture risk to a degree greater than predicted by bone density alone. Furthermore, these beneficial effects on BMD were only seen over 2 years, and it is possible that further beneficial changes in BMD, particularly at the hip, could arise beyond 2 years. The clinical significance of a $30 \%$ decrease in spine fracture risk will depend on the patients overall fracture risk based on age, gender, menopausal status, family history and history of previous fracture among other things. However, the change in likely fracture risk that we observed is probably similar to the changes seen with bisphosphonate treatment.
The implications from these findings are that current GC hormone replacement doses are still too high compared with the physiological cortisol production rate and are likely to have a negative effect on bone. In addition, single doses of more than $10 \mathrm{mg}$ hydrocortisone, e.g., the often used $15 \mathrm{mg}$ dose of $\mathrm{HC}$ in the morning, is likely to result in a very high cortisol peak in the plasma, with levels exceeding the cortisol-binding globulin-binding capacity (33) thus resulting in an abnormal high tissue exposure to the excess of unbound free cortisol.

Our data also clearly showed that PAI patients on prednisolone (mean of $5.3 \pm 0.9 \mathrm{mg} /$ day) had a significantly worse BMD compared with those on hydrocortisone (mean $22.3 \pm 4.7 \mathrm{mg}$ /day). Surprisingly they did not display any difference in BMI $(26.4 \pm 3.6$ vs $26.1 \pm$ $4.5 \mathrm{~kg} / \mathrm{m}^{2}$ respectively) suggesting that the bone is more sensitive to this long-acting synthetic GC than other tissues and a better long-time marker for GC exposure.

Our study has a number of limitations. Although this was one of the larger cohorts of patients with PAI and CAH the number of patients was still too low to examine fractures as an end point. In general the patient cohort was also heterogeneous in terms of aetiology of PAI and different forms of CAH. Furthermore, this study was not randomized. However, ethical considerations precluded a randomised design on the basis that randomized increases or decreases in $\mathrm{HC}$ dose could place patients at excessive risk (due to over- or under-treatment). Therefore, the decision to increase or decrease was individualised according to the best clinical practice guidelines.

We have therefore demonstrated for the first time that cautious reduction in hydrocortisone equivalent doses leads to increases in BMD, whereas dose increments reduced BMD. In addition, the cautious GC reduction was not associated with an increased frequency of adrenal crisis or a decrease in HRQoL. These data emphasize the need to aim for the lowest possible GC replacement dose in AI patients that maintains health and avoids the development of long-term adverse effects such as decreased BMD.

\section{Declaration of interest}

The authors declare that there is no conflict of interest that could be perceived as prejudicing the impartiality of the research reported.

\section{Funding}

This research did not receive any specific grant from any funding agency in the public, commercial or not-for-profit sector. 


\section{Author contribution statement}

M Quinkler and S Diederich designed the study. J Schulz, M Quinkler and M S Cooper wrote the manuscript. K Zopf recruited patients and organized data collection, $M$ Quinkler and $M$ Ventz recruited and treated patients. $S$ Diederich performed the DXA scans. J Schulz and K R Frey collected and analysed the data and performed statistical analysis. J Schulz, K R Frey, M S Cooper, K Zopf, M Ventz, S Diederich and M Quinkler reviewed/edited and approved the manuscript.

\section{References}

1 Esteban NV, Loughlin T, Yergey AL, Zawadzki JK, Booth JD, Winterer JC \& Loriaux DL. Daily cortisol production rate in man determuned by stable isotope dilution/mass spectrometry. Journal of Clinical Endocrinology and Metabolism 199172 39-45. (doi:10.1210/jcem-72-1-39)

2 Heureux F, Maiter D, Boutsen Y, Devogelaer JP, Jamart J \& Donckier J. Evaluation of corticosteroid replacement therapy and its effect on bones in Addison's disease. Annales d'Endocrinologie 200061 179-183.

3 Lovas K, Gjesdal CG, Christensen M, Wolff AB, Almas B, Svartberg J, Fougner KJ, Syversen U, Bollerslev J, Falch JA et al. Glucocorticoid replacement therapy and pharmacogenetics in Addison's disease: effects on bone. European Journal of Endocrinology 2009160 993-1002. (doi:10.1530/EJE-08-0880)

4 Devogelaer JP, Crabbe J \& Nagant de DC. Bone mineral density in Addison's disease: evidence for an effect of adrenal androgens on bone mass. BMJ 1987294 798-800. (doi:10.1136/bmj.294.6575.798)

5 Valero MA, Leon M, Ruiz Valdepenas MP, Larrodera L, Lopez MB, Papapietro K, Jara A \& Hawkins F. Bone density and turnover in Addison's disease: effect of glucocorticoid treatment. Bone and Mineral 199426 9-17. (doi:10.1016/S0169-6009(08)80158-4)

6 Zelissen PM, Croughs RJ, van Rijk PP \& Raymakers JA. Effect of glucocorticoid replacement therapy on bone mineral density in patients with Addison disease. Annals of Internal Medicine 1994120 207-210. (doi:10.7326/0003-4819-120-3-199402010-00005)

7 Braatvedt GD, Joyce M, Evans M, Clearwater J \& Reid IR. Bone mineral density in patients with treated Addison's disease. Osteoporosis International 199910 435-440. (doi:10.1007/s001980050251)

8 Jodar E, Valdepenas MP, Martinez G, Jara A \& Hawkins F. Long-term follow-up of bone mineral density in Addison's disease. Clinical Endocrinology 200358 617-620. (doi:10.1046/j.1365-2265.2003.01761.x)

9 Arlt W, Rosenthal C, Hahner S \& Allolio B. Quality of glucocorticoid replacement in adrenal insufficiency: clinical assessment vs. timed serum cortisol measurements. Clinical Endocrinology 200664 384-389.

10 Koetz KR, Ventz M, Diederich S \& Quinkler M. Bone mineral density is not significantly reduced in adult patients on low-dose glucocorticoid replacement therapy. Journal of Clinical Endocrinology and Metabolism 201297 85-92. (doi:10.1210/jc.2011-2036)

11 Guo CY, Weetman AP \& Eastell R. Bone turnover and bone mineral density in patients with congenital adrenal hyperplasia. Clinical Endocrinology 199645 535-541. (doi:10.1046/j.1365-2265.1996.00851.x)

12 Gussinye M, Carrascosa A, Potau N, Enrubia M, Vicens-Calvet E, Ibanez L \& Yeste D. Bone mineral density in prepubertal and in adolescent and young adult patients with the salt-wasting form of congenital adrenal hyperplasia. Pediatrics 1997100 671-674. (doi:10.1542/peds.100.4.671)

13 Mora S, Saggion F, Russo G, Weber G, Bellini A, Prinster C \& Chiumello G. Bone density in young patients with congenital adrenal hyperplasia. Bone 199618 337-340. (doi:10.1016/8756-3282(96)00003-8)

14 Stikkelbroeck NM, Oyen WJ, van der Wilt GJ, Hermus AR \& Otten BJ. Normal bone mineral density and lean body mass, but increased fat mass, in young adult patients with congenital adrenal hyperplasia. Journal of Clinical Endocrinology and Metabolism 200388 1036-1042. (doi:10.1210/jc.2002-021074)
15 Girgis R \& Winter JS. The effects of glucocorticoid replacement therapy on growth, bone mineral density, and bone turnover markers in children with congenital adrenal hyperplasia. Journal of Clinical Endocrinology and Metabolism 199782 3926-3929. (doi:10.1210/jcem. 82.12.4320)

16 Christiansen P, Molgaard C \& Muller J. Normal bone mineral content in young adults with congenital adrenal hyperplasia due to 21-hydroxylase deficiency. Hormone Research 200461 133-136. (doi:10.1159/000075588)

17 Jaaskelainen J \& Voutilainen R. Bone mineral density in relation to glucocorticoid substitution therapy in adult patients with 21-hydroxylase deficiency. Clinical Endocrinology 199645 707-713. (doi:10.1046/j.1365-2265.1996.8620871.x)

18 Hagenfeldt K, Martin RE, Ringertz H, Helleday J \& Carlstrom K. Bone mass and body composition of adult women with congenital virilizing 21-hydroxylase deficiency after glucocorticoid treatment since infancy. European Journal of Endocrinology 2000143 667-671. (doi:10.1530/eje.0. 1430667)

19 Cameron FJ, Kaymakci B, Byrt EA, Ebeling PR, Warne GL \& Wark JD. Bone mineral density and body composition in congenital adrenal hyperplasia. Journal of Clinical Endocrinology and Metabolism 199580 2238-2243.

20 de Almeida Freire PO, de Lemos-Marini SH, Maciel-Guerra AT, Morcillo AM, Matias Baptista MT, de Mello MP \& Guerra G Jr. Classical congenital adrenal hyperplasia due to 21-hydroxylase deficiency: a cross-sectional study of factors involved in bone mineral density. Journal of Bone and Mineral Metabolism 200321 396-401. (doi:10.1007/ s00774-003-0434-6)

21 King JA, Wisniewski AB, Bankowski BJ, Carson KA, Zacur HA \& Migeon CJ. Long-term corticosteroid replacement and bone mineral density in adult women with classical congenital adrenal hyperplasia. Journal of Clinical Endocrinology and Metabolism 200691 865-869. (doi:10.1210/jc.2005-0745)

22 Sciannamblo M, Russo G, Cuccato D, Chiumello G \& Mora S. Reduced bone mineral density and increased bone metabolism rate in young adult patients with 21-hydroxylase deficiency. Journal of Clinical Endocrinology and Metabolism 200691 4453-4458. (doi:10.1210/jc. 2005-2823)

23 Falhammar H, Filipsson H, Holmdahl G, Janson PO, Nordenskjold A, Hagenfeldt $\mathrm{K} \&$ Thoren M. Fractures and bone mineral density in adult women with 21-hydroxylase deficiency. Journal of Clinical Endocrinology and Metabolism 200792 4643-4649. (doi:10.1210/jc.2007-0744)

24 Zimmermann A, Sido PG, Schulze E, Al KC, Lazea C, Coldea C \& Weber MM. Bone mineral density and bone turnover in Romanian children and young adults with classical 21-hydroxylase deficiency are influenced by glucocorticoid replacement therapy. Clinical Endocrinology 200971 477-484. (doi:10.1111/j.1365-2265.2008.03518.x)

25 Husebye ES, Allolio B, Arlt W, Badenhoop K, Bensing S, Betterle C, Falorni A, Gan EH, Hulting AL, Kasperlik-Zaluska A et al. Consensus statement on the diagnosis, treatment and follow-up of patients with primary adrenal insufficiency. Journal of Internal Medicine 2014275 104-115. (doi:10.1111/joim.12162)

26 Auchus RJ \& Arlt W. Approach to the patient: the adult with congenital adrenal hyperplasia. Journal of Clinical Endocrinology and Metabolism 201398 2645-2655. (doi:10.1210/jc.2013-1440)

27 Grossman A, Johannsson G, Quinkler M \& Zelissen P. Therapy of endocrine disease: Perspectives on the management of adrenal insufficiency: clinical insights from across Europe. European Journal of Endocrinology 2013169 R165-R175. (doi:10.1530/EJE-13-0450)

28 Rivkees SA \& Crawford JD. Dexamethasone treatment of virilizing congenital adrenal hyperplasia: the ability to achieve normal growth. Pediatrics 2000106 767-773. (doi:10.1542/peds.106.4.767)

29 Caldato MC, Fernandes VT \& Kater CE. One-year clinical evaluation of single morning dose prednisolone therapy for 21-hydroxylase deficiency. Arquivos Brasileiros de Endocrinologia e Metabologia 200448 705-712. (doi:10.1590/S0004-27302004000500017) 
30 Lovas K, Curran S, Oksnes M, Husebye ES, Huppert FA \& Chatterjee VK. Development of a disease-specific Quality of Life Questionnaire in Addison's disease. Journal of Clinical Endocrinology and Metabolism 2010 95 545-551. (doi:10.1210/jc.2009-1711)

31 Oksnes M, Bensing S, Hulting AL, Kampe O, Hackemann A, Meyer G, Badenhoop K, Betterle C, Parolo A, Giordano R et al. Quality of life in European patients with Addison's disease: validity of the disease-specific questionnaire AddiQoL. Journal of Clinical Endocrinology and Metabolism 201297 568-576. (doi:10.1210/jc.2011-1901)
32 Marshall D, Johnell O \& Wedel H. Meta-analysis of how well measures of bone mineral density predict occurrence of osteoporotic fractures. BMJ 1996312 1254-1259. (doi:10.1136/ bmj.312.7041.1254)

33 Behan LA, Rogers B, Hannon MJ, O'Kelly P, Tormey W, Smith D, Thompson CJ \& Agha A. Optimizing glucocorticoid replacement therapy in severely adrenocorticotropin-deficient hypopituitary male patients. Clinical Endocrinology 201175 505-513. (doi:10.1111/j.1365-2265.2011.04074.x)

Received 7 November 2015

Revised version received 20 January 2016

Accepted 25 January 2016 